\title{
Cardinal, Median Value, Variance and Covariance of Exponential Fuzzy Numbers with Shape Function and its Applications in Ranking Fuzzy Numbers
}

\author{
S. $\operatorname{Rezvani}^{1}{ }^{1}$ \\ ${ }^{1}$ Department of Mathematics, Imam Khomaini Mritime University of Nowshahr, \\ Nowshahr, Iran \\ E-mail: salim_rezvani@yahoo.com
}

Received 11 October 2014

Accepted 29 October 2015

\begin{abstract}
In this paper, the researcher proposed a method to cardinal, median value, variance and covariance of exponential fuzzy numbers with shape function. The covariance used in this method is obtained from the exponential trapezoidal fuzzy number, first by finding mathematical expectation and then calculating the variance of each exponential fuzzy numbers by $E(A), E^{2}(A)$ and then finding the possibilistic covariance between fuzzy numbers $A$ and $B$. We are going to utilize Dubois and another researcher dominance possibility and necessity indices, within a exponential fuzzy numbers with shape function and its applications, in the case of measure of possibilistic correlation between fuzzy numbers $A$ and $B$ by their ranking possibility of their interaction compared to their possibilistic fuzzy number. Finally, we proposed a new method for ranking exponential fuzzy numbers with possibilistic variance between fuzzy numbers $A$ and $B$. This approach helps avoiding any approximation that may exist due to incorrect comparing between fuzzy numbers and can effectively rank various fuzzy numbers, their images and overcome the shortcomings of the previous techniques and also the proposed approach is very simple and easy to apply in the real life problems. For the validation, the results of the proposed approach are compared with different existing approaches.
\end{abstract}

Keywords: Covariance, Exponential Fuzzy Numbers, Mathematical expectation, Possibilistic Mean Value, Ranking Fuzzy Number, Variance.

\section{Introduction}

Comparison of fuzzy numbers is considered one of the most important topics in fuzzy logic theory. In 1965, Zadeh [25] introduced the concept of fuzzy set theory to meet those problems. The early and most important work in the field of comparing fuzzy numbers has been presented by Dubois and Prade [10]. In most of cases in our life, the data obtained for decision making are only approximately known. Fuzzy numbers allow us to make the mathematical model of linguistic variable or fuzzy environment. In addition to a fuzzy environment, ranking is a very important decision making procedure. On the other hand, the dominance possibility indices, which have been introduced by Dubois and Prade, were utilized in the field of fuzzy mathematical programming and the field of stochastic fuzzy mathematical programming in another researcher. In 1978, Dubois and Prade defined any of the fuzzy numbers as a fuzzy subset of the real line [11]. The graded mean integration representation of generalized fuzzy num-

\footnotetext{
* Salim Rezvani. Department of Mathematics, Imam Khomaini Mritime University of Nowshahr, Nowshahr, Iran.
} 
ber was introduced, by Chen and Hsieh [5], it also had been compared with some other different methods for representation with several different representation methods. ranking fuzzy values with satisfaction function investigated by Lee et al. [15]. Probability distributions can be interpreted as carriers of incomplete information [13], and possibility distributrions can be interpreted as carriers of imprecise information. In 1987 Dubois and Prade [11] defined an interval-valued expectation of fuzzy numbers, viewing them as consonant random sets. They also showed that this expectation remains additive in the sense of addition of fuzzy numbers. In 2005 Carlsson, Fuller and Majlender [3] introduced a measure of possibilistic correlation between fuzzy numbers A and B by their joint possibility distribution $\mathrm{C}$ as an average measure of their interaction (introduced earlier by Fuller and Majlender in [12]) compared to their respective marginal variances. C. Carlsson [8] introduced possibilistic mean value, variance, covariance and correlation of fuzzy numbers. S. Rezvani in [16,23] introduced correct ordering of generalized and normal trapezoidal fuzzy numbers in divergence and Median Value. In 2015 Rezvani defined a ranking fuzzy numbers for variance as values are calculated by finding expected values using the probability density function corresponding to the membership functions of the given fuzzy number and provides the correct ordering of exponential trapezoidal fuzzy numbers [24]. Moreover, S. Rezvani ([16]-[24]) evaluated the system of ranking fuzzy numbers.

This ranking used in these field was based on formulating a possibility function, whether in the case of trapezoidal fuzzy numbers. The researcher proposed a method to cardinal, median value, variance and covariance of exponential fuzzy numbers with shape function. The covariance used in this method is obtained from the exponential trapezoidal fuzzy number, first by finding mathematical expectation and then calculating the variance of each exponential fuzzy numbers by $E(A), E^{2}(A)$ and then finding the possibilistic covariance between fuzzy numbers $A$ and $B$. Finally, we proposed a new method for ranking exponential fuzzy numbers with possibilistic variance between fuzzy numbers $A$ and $B$. This method can effectively rank various fuzzy numbers, their images and overcome the shortcomings of the previous techniques and also the proposed approach is very simple and easy to apply in the real life problems. For the validation, the results of the proposed approach are compared with different existing approaches.

\section{Preliminaries}

Generally, a generalized fuzzy number $A$ is described as any fuzzy subset of the real line $R$, whose membership function $\mu_{A}$ satisfies the following conditions,

(i) $\mu_{A}$ is a continuous mapping from $R$ to the closed interval $[0,1]$,

(ii) $\mu_{A}(x)=0,-\infty<u \leqslant a$,

(iii) $\mu_{A}(x)=L(x)$ is strictly increasing on $[a, b]$,

(iv) $\mu_{A}(x)=w, b \leqslant x \leqslant c$,

(v) $\mu_{A}(x)=R(x)$ is strictly decreasing on $[c, d]$,

(vi) $\mu_{A}(x)=0, d \leqslant x<\infty$

Where $0<w \leqslant 1$ and $a, b, c$, and $d$ are real numbers. We call this type of generalized fuzzy number a trapezoidal fuzzy number, and it is denoted by $A=(a, b, c, d ; w)_{L R}$.

When $w=1$, this type of generalized fuzzy number is called normal fuzzy number and is represented by $A=(a, b, c, d)_{L R}$.

However, these fuzzy numbers always have a fix range as $[c, d]$. A exponential fuzzy number $A$ with shape function:

$$
f_{A}(x)= \begin{cases}w\left[e^{-[(b-x) /(b-a)]}\right]^{n} & a \leqslant x \leqslant b, \\ w & b \leqslant x \leqslant c, \\ w\left[e^{-[(x-c) /(d-c)]}\right]^{n} & c \leqslant x \leqslant d,\end{cases}
$$


where $0<w \leqslant 1, a, b$ are real numbers, and $c, d$ are positive real numbers. We denote this type of generalized exponential fuzzy number as $A=(a, b, c, d ; w)_{E}$. Especially, when $w=1$, we denote it as $A=(a, b, c, d)_{E}$.

we define the representation of generalized exponential fuzzy number based on the integral value of graded mean h-level as follow. Let the generalized exponential fuzzy number $A=(a, b, c, d)_{E}$, where $0<w \leqslant 1$, and $c, d$ are positive real numbers, $a, b$ are real numbers as in formula (1). Now, let two monotonic shape functions be

$$
\begin{gathered}
L(x)=w\left[e^{-[(b-x) /(b-a)]}\right]^{n}, \\
R(x)=w\left[e^{-[(x-c) /(d-c)]}\right]^{n}
\end{gathered}
$$

\section{Possibilistic Mean Value of Exponential Fuzzy Numbers}

In this section some important results, that are useful for the proposed methods, are proved.

Definition 1. [25]. Cardinality of a fuzzy number $A$ is the value of the integral

$$
\operatorname{card} A=\int_{a}^{b} A d x=\int_{0}^{1}\left(b_{\alpha}-a_{\alpha}\right) d \alpha
$$

Now, we use of above definition in exponential trapezoidal fuzzy numbers.

Theorem 1. Cardinality of a exponential trapezoidal fuzzy number $A$ characterized by (1) is the value of the integral

$$
\begin{gathered}
\operatorname{card} A=\frac{w}{n}(b-a+d-c) \\
-\frac{w}{n}\left[(b-a) e^{-n}+(d-c) e^{-n}\right]+w(c-b)
\end{gathered}
$$

\section{Proof:}

$$
\begin{gathered}
\operatorname{card} A=\int_{a}^{b} A d x \\
=\int_{a}^{b} w\left[e^{-[(b-x) /(b-a)]}\right]^{n} d x+\int_{b}^{c} w d x
\end{gathered}
$$

$$
\begin{gathered}
+\int_{c}^{d} w\left[e^{-[(x-c) /(d-c)]}\right]^{n} d x \\
=\frac{w(b-a)}{n}\left[1-e^{-n}\right]+w(c-b)+\frac{w(d-c)}{n}\left[1-e^{-n}\right] \\
=\frac{w}{n}(b-a+d-c)-\frac{w}{n}\left[(b-a) e^{-n}+(d-c) e^{-n}\right] \\
+w(c-b) .
\end{gathered}
$$

The median value of a fuzzy set $A$ was introduced as a possible scalar representative value of $A$.

Definition 2. [4]. The median value of a fuzzy number $A$ characterized by (1) is the real number $m_{A}$ from the support of $A$ such that

$$
\int_{a}^{m_{A}} A d x=\int_{m_{A}}^{d} A d x
$$

For practical purposes expression (5) can be rewritten as

$$
\int_{a}^{m_{A}} A d x=0.5 \operatorname{card} A
$$

The article can classify fuzzy numbers with respect to the "distribution" of their cardinality as follows: a fuzzy number $A$ is called

(I) a fuzzy number with equally heavy tails if $\int_{a}^{b} A d x=\int_{c}^{d} A d x$

(II) a fuzzy number with light tails if max $\left\{\int_{a}^{b} A d x, \int_{c}^{d} A d x\right\} \leqslant 0.5 \int_{a}^{d} A d x$,

(III) a fuzzy number with heavy left tail if $\int_{a}^{b} A d x>$ $0.5 \int_{a}^{d} A d x$,

(IV) a fuzzy number with heavy right tail if $\int_{c}^{d} A d x>0.5 \int_{a}^{d} A d x$.

Now the article will study location of the median value $m_{A}$ in the support of $A$. The article will also 
identify the fuzziness of $m_{A}$ determined by its membership grade $A\left(m_{A}\right)$.

Theorem 2. If $A$ is a exponential fuzzy number with light tails then

$$
\begin{aligned}
m_{A}= & \frac{w}{2}(b+c)+\frac{w}{2 n}(d-c-b+a) \\
& +\frac{w}{2 n}\left[(b-a) e^{-n}-(d-c) e^{-n}\right]
\end{aligned}
$$

and $A\left(m_{A}\right)=1$.

Proof:

$$
\begin{gathered}
m_{A}=\frac{w}{2}(b+c)+0.5\left(\int_{c}^{d} A(x) d x-\int_{a}^{b} A(x) d x\right) \\
=\frac{w}{2}(b+c)+0.5\left(\int_{c}^{d} w\left[e^{-[(x-c) /(d-c)]}\right]^{n} d x\right. \\
\left.-\int_{a}^{b}\left[e^{-[(b-x) /(b-a)]}\right]^{n} d x\right)=\frac{w}{2}(b+c) \\
+0.5\left(\frac{w(d-c)}{n}\left[1-e^{-n}\right]-\frac{w(b-a)}{n}\left[1-e^{-n]}\right)\right. \\
+\frac{w}{2 n}(b+c)+\frac{w}{2 n}(d-c-b+a) \\
\left.+(b-a) e^{-n}-(d-c) e^{-n}\right] .
\end{gathered}
$$

Corollary 1. If $A$ has equally heavy tails then $m_{A}=\frac{w}{2}(b+c)$ and $A\left(m_{A}\right)=1$.

Theorem 3. The median value of a exponential fuzzy number $A$ characterized by (1) is

$$
\begin{gathered}
\int_{a}^{m_{A}} A d x=\frac{w}{2 n}(b-a+d-c) \\
-\frac{w}{2 n}\left[(b-a) e^{-n}+(d-c) e^{-n}\right]+\frac{w}{2}(c-b)
\end{gathered}
$$

Proof: With use of theorem 1.,

$$
\begin{gathered}
\int_{a}^{m_{A}} A d x=0.5 \operatorname{car} d A \\
=\frac{1}{2}\left[\frac{w}{n}(b-a+d-c)-\frac{w}{n}\left[(b-a) e^{-n}+(d-c) e^{-n}\right]\right. \\
+w(c-b)] \\
=\frac{w}{2 n}(b-a+d-c)-\frac{w}{2 n}\left[(b-a) e^{-n}+(d-c) e^{-n}\right] \\
+\frac{w}{2}(c-b) .
\end{gathered}
$$

\section{Covariance of Exponential Fuzzy Numbers}

Definition 3. [26] The random variable $\xi$ has absolutely continuous distribution $F_{\xi}(x)$, if there a function of density for this distribution, i.e.

$$
\exists f_{\xi}(u) \geqslant 0: F_{\xi}(x)=\int_{-\infty}^{x} f_{\xi}(u) d u
$$

Properties of density:

(i). $f_{\xi}(x)=F_{\xi}^{\prime}(x)$,

(ii). $\int_{-\infty}^{\infty} f_{\xi}(u) d u=1$.

The most common examples of absolutely continuous distribution are the normal, uniform and exponential distributions.

The important numerical property of the random variable is the mathematical expectation.

Definition 4. [26] In discrete case mathematical expectation of random variable is equal to sum of the products of probabilities of random variable and its values in each element of the space, i.e.:

$$
E=\sum_{w \in \Omega} p(w) * \xi(w) .
$$

And for the absolutely continuous distribution the mathematical expectation is equal to integral over 
the space of density functions multiplied by the value of a random variable, i.e.

$$
E=\int_{x \in \Omega} x f(x) d x .
$$

Theorem 4. The mathematical expectation of a exponential fuzzy number $A$ characterized by (1) is

$$
\begin{gathered}
E(A)=\frac{w\left(e^{-n}-1\right)}{n^{2}}\left[(b-a)^{2}-(d-c)^{2}\right] \\
+\frac{w}{n}\left[(b-a)\left(b-a e^{-n}\right)+(d-c)\left(c-d e^{-n}\right)\right] \\
+\frac{w\left(c^{2}-b^{2}\right)}{2}
\end{gathered}
$$

\section{Proof:}

$$
\begin{gathered}
E(A)=\int_{-\infty}^{\infty} x A d x \\
=\int_{a}^{b} w x\left[e^{-[(b-x) /(b-a)]}\right]^{n} d x+\int_{b}^{c} w x d x \\
+\int_{c}^{d} w x\left[e^{-[(x-c) /(d-c)]}\right]^{n} d x \\
=\left[\frac{w(b-a)}{n}\left(b-a e^{-n}\right)+\frac{w(b-a)^{2}}{n^{2}}\left(e^{-n}-1\right)\right] \\
+\frac{w\left(c^{2}-b^{2}\right)}{2} \\
\left.+\frac{w(d-c)^{2}}{n^{2}}\left(1-e^{-n}\right)+\frac{w(d-c)}{n}\left(c-d e^{-n}\right)\right] \\
=\frac{w\left(e^{-n}-1\right)}{n^{2}}\left[(b-a)^{2}-(d-c)^{2}\right] \\
{\left[(b-a)\left(b-a e^{-n}\right)+(d-c)\left(c-d e^{-n}\right)\right]}
\end{gathered}
$$

$$
+\frac{w\left(c^{2}-b^{2}\right)}{2} .
$$

Definition 5. Let $A$ be fuzzy number. We define the variance of $A$ by

$$
\operatorname{Var}(A)=E(x-E(x))^{2}=E\left(x^{2}\right)-E^{2}(x)
$$

Theorem 5. let $A$ be fuzzy number. Then

$$
\begin{gathered}
\operatorname{Var}(A)=\left[\frac{w}{n}(b-a+d-c)\left(b^{2}+c^{2}-e^{-n}\left(a^{2}+d^{2}\right)\right)\right. \\
+\frac{2 w\left(1-e^{-n}\right)}{n^{3}}\left((d-c)^{3}+(b-a)^{3}\right) \\
\left.+\frac{2 w}{n^{2}}\left((d-c)^{2}\left(c-d e^{-n}\right)+(b-a)^{2}\left(a e^{-n}-b\right)\right)\right] \\
-\left[\frac{w\left(e^{-n}-1\right)}{n^{2}}\left[(b-a)^{2}-(d-c)^{2}\right]\right. \\
+\frac{w}{n}\left[(b-a)\left(b-a e^{-n}\right)+(d-c)\left(c-d e^{-n}\right)\right] \\
\left.+\frac{w\left(c^{2}-b^{2}\right)}{2}\right]^{2}
\end{gathered}
$$

Proof: We know of equ.(12) that

$$
\begin{gathered}
E(x)=\frac{w\left(e^{-n}-1\right)}{n^{2}}\left[(b-a)^{2}-(d-c)^{2}\right] \\
+\frac{w}{n}\left[(b-a)\left(b-a e^{-n}\right)+(d-c)\left(c-d e^{-n}\right)\right] \\
+\frac{w\left(c^{2}-b^{2}\right)}{2}
\end{gathered}
$$

So we should obtain $E\left(x^{2}\right)$,

$$
\begin{gathered}
E\left(x^{2}\right)=\int_{-\infty}^{\infty} x^{2} A d x \\
=\int_{a}^{b} w x^{2}\left[e^{-[(b-x) /(b-a)]}\right]^{n} d x+\int_{b}^{c} w x^{2} d x
\end{gathered}
$$




$$
\begin{aligned}
& +\int_{c}^{d} w x^{2}\left[e^{-[(x-c) /(d-c)]}\right]^{n} d x \\
& =\left[\frac{w(b-a)}{n}\left(b^{2}-a^{2} e^{-n}\right)\right. \\
& \left.-\frac{2(b-a)}{n}\left[\frac{w(b-a)}{n}\left(b-a e^{-n}\right)+\frac{w(b-a)^{2}}{n^{2}}\left(e^{-n}-1\right)\right]\right] \\
& +\frac{w\left(c^{3}-b^{3}\right)}{3} \\
& +\left[\frac{w(d-c)}{n}\left(c^{2}-d^{2} e^{-n}\right)\right. \\
& +\frac{2(d-c)}{n}\left[\frac{w(d-c)^{2}}{n^{2}}\left(1-e^{-n}\right)\right. \\
& \left.\left.+\frac{w(d-c)}{n}\left(c-d e^{-n}\right)\right]\right] \\
& =\frac{w}{n}(b-a+d-c)\left(b^{2}+c^{2}-e^{-n}\left(a^{2}+d^{2}\right)\right) \\
& +\frac{2 w\left(1-e^{-n}\right)}{n^{3}}\left((d-c)^{3}+(b-a)^{3}\right) \\
& +\frac{2 w}{n^{2}}\left((d-c)^{2}\left(c-d e^{-n}\right)+(b-a)^{2}\left(a e^{-n}-b\right)\right)
\end{aligned}
$$

So

$$
\begin{gathered}
E\left(x^{2}\right)=\frac{w}{n}(b-a+d-c)\left(b^{2}+c^{2}-e^{-n}\left(a^{2}+d^{2}\right)\right) \\
+\frac{2 w\left(1-e^{-n}\right)}{n^{3}}\left((d-c)^{3}+(b-a)^{3}\right) \\
+\frac{2 w}{n^{2}}\left((d-c)^{2}\left(c-d e^{-n}\right)+(b-a)^{2}\left(a e^{-n}-b\right)\right)
\end{gathered}
$$

Therefore with use equs((15),(16)) we have

$$
\begin{gathered}
\operatorname{Var}(A)=E(x-E(x))^{2}=E\left(x^{2}\right)-E^{2}(x) \\
=\frac{w}{n}(b-a+d-c)\left(b^{2}+c^{2}-e^{-n}\left(a^{2}+d^{2}\right)\right) \\
+\frac{2 w\left(1-e^{-n}\right)}{n^{3}}\left((d-c)^{3}+(b-a)^{3}\right) \\
+\frac{2 w}{n^{2}}\left((d-c)^{2}\left(c-d e^{-n}\right)+(b-a)^{2}\left(a e^{-n}-b\right)\right) \\
-\left\{\frac{w\left(e^{-n}-1\right)}{n^{2}}\left[(b-a)^{2}-(d-c)^{2}\right]\right. \\
\left.\left.+(d-c)\left(c-d e^{-n}\right)\right]+\frac{w\left(c^{2}-b^{2}\right)}{2}\right\}^{2} \\
+\frac{w}{n}\left[(b-a)\left(b-a e^{-n}\right)\right.
\end{gathered}
$$

Definition 6. The covariance between fuzzy numbers $A$ and $B$ is defined as

$$
\operatorname{Cov}(x, y)=E(x . y)-E(x) E(y)
$$

Theorem 6. let $A$ be exponential fuzzy number. Then

$$
\operatorname{Cov}(A, A)=\operatorname{Var}(A)
$$

Proof: Let $A=(a, b, c, d)_{E}$, A exponential fuzzy number with shape function is

$$
f_{A}(x)= \begin{cases}w\left[e^{-[(b-x) /(b-a)]}\right]^{n} & a \leqslant x \leqslant b \\ w & b \leqslant x \leqslant c \\ w\left[e^{-[(x-c) /(d-c)]}\right]^{n} & c \leqslant x \leqslant d\end{cases}
$$

From (17) we deduce

$$
\operatorname{Cov}(A, A)=E(A . A)-E(A) E(A)
$$

first $E(A . A)$,

$$
E(A . A)=\int_{-\infty}^{\infty} x . x A d x=\int_{-\infty}^{\infty} x^{2} A d x=E\left(x^{2}\right)
$$


Of (16) we know

$$
\begin{gathered}
E\left(x^{2}\right)=\frac{w}{n}(b-a+d-c)\left(b^{2}+c^{2}-e^{-n}\left(a^{2}+d^{2}\right)\right) \\
+\frac{2 w\left(1-e^{-n}\right)}{n^{3}}\left((d-c)^{3}+(b-a)^{3}\right) \\
+\frac{2 w}{n^{2}}\left((d-c)^{2}\left(c-d e^{-n}\right)+(b-a)^{2}\left(a e^{-n}-b\right)\right),
\end{gathered}
$$

Now $E(A) E(A)$,

$$
E(A) E(A)=E^{2}(A)
$$

Of (15) we know

$$
\begin{gathered}
E(x)=\frac{w\left(e^{-n}-1\right)}{n^{2}}\left[(b-a)^{2}-(d-c)^{2}\right] \\
+\frac{w}{n}\left[(b-a)\left(b-a e^{-n}\right)+(d-c)\left(c-d e^{-n}\right)\right] \\
+\frac{w\left(c^{2}-b^{2}\right)}{2}
\end{gathered}
$$

So we deduce

$$
\begin{gathered}
E^{2}(x)=\left\{\frac{w\left(e^{-n}-1\right)}{n^{2}}\left[(b-a)^{2}-(d-c)^{2}\right]\right. \\
+\frac{w}{n}\left[(b-a)\left(b-a e^{-n}\right)+(d-c)\left(c-d e^{-n}\right)\right] \\
\left.+\frac{w\left(c^{2}-b^{2}\right)}{2}\right\}^{2}
\end{gathered}
$$

Putting value of (20) and (21) in (19) yields

$\operatorname{Cov}(A, A)=\frac{w}{n}(b-a+d-c)\left(b^{2}+c^{2}-e^{-n}\left(a^{2}+d^{2}\right)\right)$

$$
+\frac{2 w\left(1-e^{-n}\right)}{n^{3}}\left((d-c)^{3}+(b-a)^{3}\right)
$$

$$
\begin{aligned}
& +\frac{2 w}{n^{2}}\left((d-c)^{2}\left(c-d e^{-n}\right)+(b-a)^{2}\left(a e^{-n}-b\right)\right) \\
& \quad-\left\{\frac{w\left(e^{-n}-1\right)}{n^{2}}\left[(b-a)^{2}-(d-c)^{2}\right]\right. \\
& \left.+\frac{w}{n}\left[(b-a)\left(b-a e^{-n}\right)+(d-c)\left(c-d e^{-n}\right)\right]+\frac{w\left(c^{2}-b^{2}\right)}{2}\right\}^{2} \\
& \qquad=E\left(x^{2}\right)-E^{2}(x)=\operatorname{Var}(A) . \\
& \text { So } \operatorname{Cov}(A, A)=\operatorname{Var}(A) .
\end{aligned}
$$

Corollary 2. let $A, B$ and $C$ be three exponential trapezoidal fuzzy number and let $\lambda, \mu \in[0,1]$, Then the following properties hold,

(i) $\operatorname{Cov}(A, B)=\operatorname{Cov}(B, A)$,

(ii) $\operatorname{Cov}(A, \lambda+\mu A)=\mu \operatorname{Var}(A)$,

(iii) $\operatorname{Cov}(\lambda A+\mu B, C)=\lambda \operatorname{Cov}(A, C)+\mu \operatorname{Cov}(B, C)$.

Theorem 7. let $A$ and $B$ be two exponential trapezoidal fuzzy number and let $\lambda, \mu \in[0,1]$, then

$$
\begin{aligned}
\operatorname{Var}(\lambda A+\mu B) & =\lambda^{2} \operatorname{Var}(A)+\mu^{2} \operatorname{Var}(B) \\
+ & +2 \lambda \mu \operatorname{Cov}(A, B)
\end{aligned}
$$

Proof: From (18) we deduce

$$
\operatorname{Var}(\lambda A+\mu B)=\operatorname{Cov}(\lambda A+\mu B, \lambda A+\mu B)
$$

Therefore

$$
\begin{gathered}
\operatorname{Cov}(\lambda A+\mu B, \lambda A+\mu B)=\operatorname{Cov}(\lambda A, \lambda A) \\
+\operatorname{Cov}(\lambda A, \mu B) \\
+\operatorname{Cov}(\mu B, \lambda A)+\operatorname{Cov}(\mu B, \mu B)
\end{gathered}
$$

To prove Theorem 7 , we need the following.

(i) $\operatorname{Cov}(\lambda A, \lambda A)$,(ii) $\operatorname{Cov}(\lambda A, \mu B)$,

(iii) $\operatorname{Cov}(\mu B, \lambda A)$, (iv) $\operatorname{Cov}(\mu B, \mu B)$. 
(i) $\operatorname{Cov}(\lambda A, \lambda A)=\operatorname{Var}(\lambda A)=E\left(\lambda^{2} A^{2}\right)-(E(\lambda A))^{2}$

$$
\Rightarrow E\left(\lambda^{2} A^{2}\right)-(E(\lambda A))^{2}
$$

$$
E(\lambda A)=\int_{-\infty}^{\infty} x \lambda A d x=
$$

$=\int_{a}^{b} w x \lambda\left[e^{-[(b-x) /(b-a)]}\right]^{n} d x+\int_{b}^{c} w x \lambda d x$

$$
+\int_{c}^{d} w x \lambda\left[e^{-[(x-c) /(d-c)]}\right]^{n} d x
$$

$$
=\frac{\lambda w\left(e^{-n}-1\right)}{n^{2}}\left[(b-a)^{2}-(d-c)^{2}\right]
$$

$+\frac{\lambda w}{n}\left[(b-a)\left(b-a e^{-n}\right)+(d-c)\left(c-d e^{-n}\right)\right]$

$$
+\frac{\lambda w\left(c^{2}-b^{2}\right)}{2} .
$$

$$
E\left(\lambda^{2} x^{2}\right)=\int_{-\infty}^{\infty} \lambda^{2} x^{2} A d x
$$

$=\int_{a}^{b} w \lambda^{2} x^{2}\left[e^{-[(b-x) /(b-a)]}\right]^{n} d x+\int_{b}^{c} w \lambda^{2} x^{2} d x$

$$
+\int_{c}^{d} w \lambda^{2} x^{2}\left[e^{-[(x-c) /(d-c)]}\right]^{n} d x
$$

$E\left(\lambda^{2} x^{2}\right)=\frac{\lambda^{2} w}{n}(b-a+d-c)\left(b^{2}+c^{2}-e^{-n}\left(a^{2}+d^{2}\right)\right)$

$$
+\frac{2 \lambda^{2} w\left(1-e^{-n}\right)}{n^{3}}\left((d-c)^{3}+(b-a)^{3}\right)
$$

$+\frac{2 \lambda^{2} w}{n^{2}}\left((d-c)^{2}\left(c-d e^{-n}\right)+(b-a)^{2}\left(a e^{-n}-b\right)\right)$

$$
\begin{gathered}
=\left[\frac{\lambda^{2} w}{n}(b-a+d-c)\left(b^{2}+c^{2}-e^{-n}\left(a^{2}+d^{2}\right)\right)\right. \\
\left.+\frac{2 \lambda^{2} w\left(1-e^{-n}\right)}{n^{3}}\left((d-c)^{3}+(b-a)^{3}\right)\right] \\
-\left[\frac{\lambda w\left(e^{-n}-1\right)}{n^{2}}\left[(b-a)^{2}-(d-c)^{2}\right]\right.
\end{gathered}
$$$$
+\frac{\lambda w}{n}\left[(b-a)\left(b-a e^{-n}\right)+(d-c)\left(c-d e^{-n}\right)\right]
$$$$
\left.+\frac{\lambda w\left(c^{2}-b^{2}\right)}{2}\right]^{2}
$$

$$
\begin{gathered}
=\lambda^{2}\left[\frac{w}{n}(b-a+d-c)\left(b^{2}+c^{2}-e^{-n}\left(a^{2}+d^{2}\right)\right)\right. \\
\left.+\frac{2 w\left(1-e^{-n}\right)}{n^{3}}\left((d-c)^{3}+(b-a)^{3}\right)\right] \\
-\lambda^{2}\left[\frac{w\left(e^{-n}-1\right)}{n^{2}}\left[(b-a)^{2}-(d-c)^{2}\right]\right.
\end{gathered}
$$

$$
\begin{aligned}
& \left.+\frac{w}{n}\left[(b-a)\left(b-a e^{-n}\right)+(d-c)\left(c-d e^{-n}\right)\right]+\frac{w\left(c^{2}-b^{2}\right)}{2}\right]^{2} \\
& =\lambda^{2}\left(\left[\frac{w}{n}(b-a+d-c)\left(b^{2}+c^{2}-e^{-n}\left(a^{2}+d^{2}\right)\right)\right.\right.
\end{aligned}
$$

$$
\begin{aligned}
& \left.+\frac{2 w\left(1-e^{-n}\right)}{n^{3}}\left((d-c)^{3}+(b-a)^{3}\right)\right] \\
& -\left[\frac{w\left(e^{-n}-1\right)}{n^{2}}\left[(b-a)^{2}-(d-c)^{2}\right]\right.
\end{aligned}
$$

$$
+\frac{w}{n}\left[(b-a)\left(b-a e^{-n}\right)+(d-c)\left(c-d e^{-n}\right)\right]
$$




$$
\begin{gathered}
\left.\left.+\frac{w\left(c^{2}-b^{2}\right)}{2}\right]^{2}\right) \\
=\lambda^{2}\left(E\left(A^{2}\right)-E^{2}(A)\right)=\lambda^{2} \operatorname{Var}(A)
\end{gathered}
$$

So

$$
\text { (i) } \begin{aligned}
\operatorname{Cov}(\lambda A, \lambda A) & =\lambda^{2}\left(E\left(A^{2}\right)-E^{2}(A)\right) \\
= & \lambda^{2} \operatorname{Var}(A)
\end{aligned}
$$

The same way, we deduce

(iv) $\operatorname{Cov}(\mu B, \mu B)=\operatorname{Var}(\mu B)=E\left(\mu^{2} B^{2}\right)-(E(\mu B))^{2}$

$$
=\mu^{2}\left(E\left(B^{2}\right)-E^{2}(B)\right)=\mu^{2} \operatorname{Var}(B)
$$

Now, prove for (ii) $\operatorname{Cov}(\lambda A, \mu B)$,

From (17) we deduce

$$
\begin{gathered}
\operatorname{Cov}(\lambda A, \mu B)=E(\lambda A . \mu B)-E(\lambda A) E(\mu B) \\
=\lambda \mu E(A . B)-\lambda E(A) \mu E(B) \\
=\lambda \mu E(A . B)-\lambda \mu E(A) E(B) \\
=\lambda \mu(E(A . B)-E(A) E(B))=\lambda \mu \operatorname{Cov}(A, B)
\end{gathered}
$$

So

$$
\text { (ii) } \operatorname{Cov}(\lambda A, \mu B)=\lambda \mu \operatorname{Cov}(A, B)
$$

The same way, we deduce

$$
\begin{aligned}
& \operatorname{Cov}(\mu B, \lambda A)=\mu \lambda E(B . A)-\mu \lambda E(B) E(A) \\
= & \mu \lambda(E(B . A)-E(B) E(A))=\mu \lambda \operatorname{Cov}(B, A)
\end{aligned}
$$

By using of Corollary 2.(first properties), we know $\operatorname{Cov}(B, A)=\operatorname{Cov}(A, B)$, Then

$$
\text { (iii) } \operatorname{Cov}(\mu B, \lambda A)=\lambda \mu \operatorname{Cov}(A, B)
$$

Putting (25), (26), (27) and (28) in (24) yields

$$
\begin{gathered}
\operatorname{Cov}(\lambda A+\mu B, \lambda A+\mu B)=\lambda^{2} \operatorname{Var}(A)+\mu^{2} \operatorname{Var}(B) \\
+\lambda \mu \operatorname{Cov}(A, B)+\lambda \mu \operatorname{Cov}(A, B)
\end{gathered}
$$

Putting (29) in (23) yields

$$
\begin{gathered}
\operatorname{Var}(\lambda A+\mu B) \\
=\lambda^{2} \operatorname{Var}(A)+\mu^{2} \operatorname{Var}(B)+2 \lambda \mu \operatorname{Cov}(A, B) .
\end{gathered}
$$

\section{Ranking Variance in Exponential Fuzzy Numbers}

Let $A=\left(a_{1}, b_{1}, \alpha_{1}, \beta_{1} ; w_{1}\right)$ and $B=\left(a_{2}, b_{2}, \alpha_{2}, \beta_{2} ; w_{2}\right)$ be two generalized exponential trapezoidal fuzzy number, then use the following steps to compare $A, B$

* step 1: Find $E(x)_{A}$ and $E(x)_{B}$,

* step 2: Find $E\left(x^{2}\right)_{A}$ and $E\left(x^{2}\right)_{B}$,

* step 3: Find $\operatorname{Var}(A)$ and $\operatorname{Var}(B)$,

* step 4: Use of following definition for comparing fuzzy numbers.

Definition 7. Let $A, B$ be trapezoidal fuzzy numbers, then

(i) $A>B \Leftrightarrow \operatorname{Var}(A)>\operatorname{Var}(B)$,

(ii) $A<B \Leftrightarrow \operatorname{Var}(A)<\operatorname{Var}(B)$,

(iii) $A \sim B \Leftrightarrow \operatorname{Var}(A) \sim \operatorname{Var}(B)$.

Remark 1. In this section suppose $n=1$. A fuzzy number A with shape function where $n>0$, will be denoted by $A=(a, b, \alpha, \beta ; w)_{n}$, if $n=1$, we simply write $A=(a, b, \alpha, \beta ; w)$, which is known as a trapezoidal fuzzy number. 


\subsection{Results}

In this section, seven sets of fuzzy numbers are compared using the proposed approach and existing approaches. The results are shown in Table 1.

Example 1. Let $A=(0.2,0.4,0.6,0.8 ; 0.35)$ and $B=(0.1,0.2,0.3,0.4 ; 0.7)$ be two generalized trapezoidal fuzzy number, then

* step 1: $E(x)_{A}=0.0798$ and $E(x)_{B}=0.0392$,

* step 2: $E\left(x^{2}\right)_{A}=0.0443$ and $E\left(x^{2}\right)_{B}=0.011$,

* step 3: $\operatorname{Var}(A)=0.0443-(0.0798)^{2}=0.0379$ and $\operatorname{Var}(B)=0.011-(0.0392)^{2}=0.0095$,

* step 4: $\operatorname{Var}(A)>\operatorname{Var}(B)$ then $A>B$.

Example 2. Let $A=(0.1,0.2,0.4,0.5 ; 1)$ and $B=(0.1,0.3,0.3,0.5 ; 1)$ be two generalized trapezoidal fuzzy number, then

* step 1: $E(x)_{A}=0.098$ and $E(x)_{B}=0.076$,

* step 2: $E\left(x^{2}\right)_{A}=0.0247$ and $E\left(x^{2}\right)_{B}=0.043$,

* step 3: $\operatorname{Var}(A)=0.0247-(0.098)^{2}=0.0151$ and $\operatorname{Var}(B)=0.043-(0.076)^{2}=0.0372$,

* step 4: $\operatorname{Var}(A)<\operatorname{Var}(B)$ then $A<B$.

Example 3. Let $A=(0.1,0.2,0.4,0.5 ; 1)$ and $B=(1,1,1,1 ; 1)$ be two generalized trapezoidal fuzzy number, then

* step 1: $E(x)_{A}=0.098$ and $E(x)_{B}=0$,

* step 2: $E\left(x^{2}\right)_{A}=0.0247$ and $E\left(x^{2}\right)_{B}=0$,

* step 3: $\operatorname{Var}(A)=0.0247-(0.098)^{2}=0.0151$ and $\operatorname{Var}(B)=0$,

* step 4: $\operatorname{Var}(A)>\operatorname{Var}(B)$ then $A>B$.

Example 4. Let $A=(-0.5,-0.3,-0.3,-0.1 ; 1)$ and $B=(0.1,0.3,0.3,0.5 ; 1)$ be two generalized trapezoidal fuzzy number, then

* step 1: $E(x)_{A}=-0.076$ and $E(x)_{B}=0.076$,

* step 2: $E\left(x^{2}\right)_{A}=0.043$ and $E\left(x^{2}\right)_{B}=0.043$,

* step 3: $\operatorname{Var}(A)=0.043-(-0.076)^{2}=0.0372$ and $\operatorname{Var}(B)=0.043-(0.076)^{2}=0.0372$,

* step 4: $\operatorname{Var}(A) \sim \operatorname{Var}(B)$ then $A \sim B$.

Example 5. Let $A=(0.3,0.5,0.5,1 ; 1)$ and $B=$ $(0.1,0.6,0.6,0.8 ; 1)$ be two generalized trapezoidal fuzzy number, then

* step 1: $E(x)_{A}=0.2753$ and $E(x)_{B}=0.2097$,

* step 2: $E\left(x^{2}\right)_{A}=0.2714$ and $E\left(x^{2}\right)_{B}=0.2484$,

* step 3: $\operatorname{Var}(A)=0.2714-(0.2753)^{2}=0.1954$ and $\operatorname{Var}(B)=0.2484-(0.2097)^{2}=0.2044$,

* step 4: $\operatorname{Var}(A)<\operatorname{Var}(B)$ then $A<B$.

Example 6. Let $A=(0,0.4,0.6,0.8 ; 1)$ and $B=$ $(0.2,0.5,0.5,0.9 ; 1)$ and $C=(0.1,0.6,0.7,0.8 ; 1)$ be three generalized trapezoidal fuzzy number, then

* step 1: $E(x)_{A}=0.2464$ and $E(x)_{B}=0.2411$ and $E(x)_{C}=0.2348$,

* step 2: $E\left(x^{2}\right)_{A}=0.1615$ and $E\left(x^{2}\right)_{B}=0.2247$ and $E\left(x^{2}\right)_{C}=0.253$,

* step 3: $\operatorname{Var}(A)=0.1615-(0.2464)^{2}=0.1005$ and $\operatorname{Var}(B)=0.2247-(0.2411)^{2}=0.1667$ and $\operatorname{Var}(C)=0.253-(0.2348)^{2}=0.198$,

* step 4: $\operatorname{Var}(A)<\operatorname{Var}(B)<\operatorname{Var}(C)$ then $A<B<$ C.

Example 7. Let $A=(0.1,0.2,0.4,0.5 ; 1)$ and $B=(-2,0,0,2 ; 1)$ be two generalized trapezoidal fuzzy number, then

* step 1: $E(x)_{A}=0.098$ and $E(x)_{B}=0$, 
* step 2: $E\left(x^{2}\right)_{A}=0.0247$ and $E\left(x^{2}\right)_{B}=-3.36$,

* step 3: $\operatorname{Var}(A)=0.0247-(0.098)^{2}=0.0151$ and $\operatorname{Var}(B)=-3.36-(0)^{2}=-3.36$,

* step 4: $\operatorname{Var}(A)>\operatorname{Var}(B)$ then $A>B$.

The results of example (8) are shown in Table 2.

Example 8. Consider the following sets of fuzzy sets:

Set 1: $A_{1}=(1,1,1,3), A_{2}=(1,1,1,7)$.

Set 2: $A_{1}=(2,4,4,6), A_{2}=(1,5,5,6), A_{3}=$ $(3,5,5,6)$.

Set 3: $A_{1}=(2,3,3,8), A_{2}=(2,3,7,8), A_{3}=$ $(2,3,3,10)$.

Set 4: $A_{1}=(1,5,5,5), A_{2}=(2,3,5,5)$.

Set 5: $A_{1}=(2,4,4,6), A_{2}=(1,5,5,6)$.

According to the deviation degree method, we have:

In Set 1 :

* step 1: $E(x)_{A}=0.31$ and $E(x)_{B}=7.14$,

* step 2: $E\left(x^{2}\right)_{A}=5.8$ and $E\left(x^{2}\right)_{B}=58.68$,

* step 3: $\operatorname{Var}(A)=5.8-(0.31)^{2}=5.7$ and $\operatorname{Var}(B)=$ $58.68-(7.14)^{2}=7.7$,

* step 4: $\operatorname{Var}(A)<\operatorname{Var}(B)$ then $A<B$.

In Set 2 :

* step 1: $E(x)_{A}=10.08$ and $E(x)_{B}=11.85$ and $E(x)_{C}=8.67$,

* step 2: $E\left(x^{2}\right)_{A}=69.12$ and $E\left(x^{2}\right)_{B}=60.37$ and $E\left(x^{2}\right)_{C}=80.79$,

* step 3: $\operatorname{Var}(A)=69.12-(10.08)^{2}=-95.61$ and $\operatorname{Var}(B)=60.37-(11.85)^{2}=-80.05$ and
$\operatorname{Var}(C)=80.79-(8.67)^{2}=5.62$,

* step 4: $\operatorname{Var}(A)<\operatorname{Var}(B)<\operatorname{Var}(C)$ then $A<B<$ C.

In Set 3 :

* step 1: $E(x)_{A}=17.58$ and $E(x)_{B}=26.3$ and $E(x)_{C}=27.6$,

* step 2: $E\left(x^{2}\right)_{A}=113.28$ and $E\left(x^{2}\right)_{B}=71.76$ and $E\left(x^{2}\right)_{C}=196.48$,

* step 3: $\operatorname{Var}(A)=113.28-(17.58)^{2}=-195.78$ and $\operatorname{Var}(B)=71.76-(26.3)^{2}=-619.93$ and $\operatorname{Var}(C)=196.48-(27.6)^{2}=-565.28$,

* step 4: $\operatorname{Var}(B)<\operatorname{Var}(C)<\operatorname{Var}(A)$ then $B<C<$ A.

In Set 4 :

* step 1: $E(x)_{A}=8.44$ and $E(x)_{B}=9.63$,

* step 2: $E\left(x^{2}\right)_{A}=94$ and $E\left(x^{2}\right)_{B}=20.01$,

* step 3: $\operatorname{Var}(A)=94-(8.44)^{2}=22.77$ and $\operatorname{Var}(B)=20.01-(9.63)^{2}=-72.72$,

* step 4: $\operatorname{Var}(B)<\operatorname{Var}(A)$ then $B<A$.

In Set 5 :

* step 1: $E(x)_{A}=10.08$ and $E(x)_{B}=11.85$,

* step 2: $E\left(x^{2}\right)_{A}=69.12$ and $E\left(x^{2}\right)_{B}=60.37$,

* step 3: $\operatorname{Var}(A)=69.12-(10.08)^{2}=-95.61$ and $\operatorname{Var}(B)=60.37-(11.85)^{2}=-80.05$,

* step 4: $\operatorname{Var}(A)<\operatorname{Var}(B)$ then $A<B$.

\section{Conclusion}

In this paper, we proposed a new method to ranking exponential fuzzy numbers by variance of fuzzy 
numbers. The variance used in this method is obtained from the mathematical expectation and then calculating by $E(A), E^{2}(A)$ and then finding the possibilistic variance between in ranking fuzzy numbers $A$ and $B$. The main advantage of the proposed approach is that the proposed approach provides the correct ordering of generalized and normal trapezoidal fuzzy numbers and also the proposed approach is very simple and easy to apply in the real life problems. This method can effectively rank various fuzzy numbers.

\section{References}

1. S. Abbasbandy, T. Hajjari, A new approach for ranking of trapezoidal fuzzy numbers, Computers and Mathematics with Applications, 57 (3), 413-419, (2009).

2. S. Bodjanova, Median value and median interval of a fuzzy number, Infomation Sciences, 172, 73-89, (2005).

3. C. Carlsson, R. Fuller and P. Majlender, On possibilistic correlation, Fuzzy Sets and Systems, 155, 425-445, (2005).

4. Chen, SJ., Chen, SM., Fuzzy risk analysis based on the ranking of generalized trapezoidal fuzzy numbers, Applied Intelligence, 26 (1),1-11, (2007).

5. S. Chen and Guo-Chin Li, Representation, Ranking, and Distance of Fuzzy Number with Exponential Membership Function Using Graded mean Integration method, Tamsui oxford journal of mathematical science, 16 (2), 123-131, (2000).

6. Chen, SM., Chen, JH., Fuzzy risk analysis based on ranking generalized fuzzy numbers with different heights and different spreads, Expert Systems with Applications, 36 (3), 6833-6842, (2009).

7. Cheng, C. H., A new approach for ranking fuzzy numbers by distance method, Fuzzy Sets and System, 95, 307-317, (1998).

8. Christer Carlsson and Robert Fuller, On Possibilistic Mean Value, Variance, Covariance and Correlation of Fuzzy Numbers, Towards Intelligent Engineering and Information Technology, vol. 243, pp. 17-36, (2009).

9. Chu, T. C., Tsao, C. T, Ranking fuzzy numbers with an area between the centroid point and original point, Computers and Mathematics with Applications, 43, 111-117, (2002).

10. D. Dubois and H. Prade, Ranking fuzzy numbers in the setting of possibility theory, Information Sciences 30, 183224 (1983).
11. D. Dubois and H. Prade, The mean value of a fuzzy number, Fuzzy Sets and Systems, 24, 279-300, (1987).

12. R. Fuller and P. Majlender, On interactive fuzzy numbers, Fuzzy Sets and Systems, 143, 355-369, (2004).

13. E.T. Jaynes, Probability Theory : The Logic of Science, Cambridge University Press, 2003.

14. Kumar, A., Singh P., Kaur A., Kaur, P., RM approach for ranking of generalized trapezoidal fuzzy numbers, Fuzzy Information and Engineering, 2 (1), 3747, (2010).

15. K. Lee, H. Chu, H. Lee-Kwang, Ranking fuzzy values with satisfaction function, Fuzzy Sets and Systems, 64, 295-309, (1994).

16. S. Rezvani, A New Method for Rank, Mode, Divergence and Spread on Generalized Exponential Trapezoidal Fuzzy Numbers, Turkish Journal of Fuzzy Systems, Vol.3, No.2, pp. 98-107, (2012).

17. S. Rezvani, Multiplication Operation on Trapezoidal Fuzzy Numbers, Journal of Physical Sciences, Vol. 15,17-26, (2011).

18. S. Rezvani, New Method for Ranking in Perimeters of two Generalized Trapezoidal Fuzzy Numbers, International Journal of Applied Operational Research, Vol. 2, No. 3, pp. 83-90, (2012).

19. S. Rezvani, A New Approach Ranking of Exponential Trapezoidal Fuzzy Numbers,Journal of Physical Sciences, Vol. 16, 45-57, (2012).

20. S. Rezvani, A New Method for Ranking in Areas of two Generalized Trapezoidal Fuzzy Numbers, International Journal of Fuzzy Logic Systems (IJFLS), Vol.3, No1, 17-24, (2013).

21. S. Rezvani, Ranking Generalized Trapezoidal Fuzzy Numbers with Euclidean Distance by the Incentre of Centroids, Mathematica Aeterna, Vol. 3, no. 2, 103114, (2013).

22. S. Rezvani, Ranking Method of Trapezoidal Intuitionistic Fuzzy Numbers, Annals of Fuzzy Mathematics and Informatics, Volume 5, No. 3, pp. 515-523, (2013).

23. S. Rezvani, Ranking Exponential Trapezoidal Fuzzy Numbers by Median Value, Journal of Fuzzy Set Valued Analysis, Volume 2013, Year 2013 Article ID jfsva-00139, 1-9.

24. S. Rezvani, Ranking generalized exponential trapezoidal fuzzy numbers based on variance, Applied Mathematics and Computation, 262, 191198, (2015).

25. L. A. Zadeh, Fuzzy set, Information and Control, vol.8,no.3, pp.338-353, (1965).

26. O. V. Lapko, O. O. Provotar, O. O. Provotar, Basic similarities and differences ways of description uncertain variables, taac.org.ua/files/a2012/proceedings/UA-1-Lapko285, (2012). 
Table (1): A comparison of the ranking results for different approaches

\begin{tabular}{|c|c|c|c|c|c|c|c|}
\hline Approaches & Ex.1 & Ex.2 & Ex.3 & Ex.4 & Ex.5 & Ex.6 & Ex.7 \\
\hline Cheng [7] & $A<B$ & $A \sim B$ & Error & $A \sim B$ & $A>B$ & $A<B<C$ & Error \\
\hline Chu [9] & $A<B$ & $A \sim B$ & Error & $A<B$ & $A>B$ & $A<B<C$ & Error \\
\hline Chen [4] & $A<B$ & $A<B$ & $A<B$ & $A<B$ & $A>B$ & $A<C<B$ & $A>B$ \\
\hline Abbasbandy [1] & Error & $A \sim B$ & $A<B$ & $A \sim B$ & $A<B$ & $A<B<C$ & $A>B$ \\
\hline Chen [6] & $A<B$ & $A<B$ & $A<B$ & $A<B$ & $A>B$ & $A<B<C$ & $A>B$ \\
\hline Kumar [14] & $A>B$ & $A \sim B$ & $A<B$ & $A<B$ & $A>B$ & $A<B<C$ & $A>B$ \\
\hline S. Rezvani [21] & $A>B$ & $A>B$ & $A<B$ & $A<B$ & $A<B$ & $A<B<C$ & $A>B$ \\
\hline Proposed approach & $A>B$ & $A<B$ & $A>B$ & $A \sim B$ & $A<B$ & $A<B<C$ & $A>B$ \\
\hline
\end{tabular}

Table (2): The results of ranking fuzzy numbers in Example 8.

\begin{tabular}{|c|c|c|c|c|c|c|}
\hline Authors & Fuzzy number & Set 1 & Set 2 & Set 3 & Set 4 & Set 5 \\
\hline Wang et al.2009 & $A_{1}$ & 0 & 0 & 0 & 0 & 0.792 \\
& $A_{2}$ & 0 & 0 & 0.444 & 1.100 & 0.784 \\
& $A_{3}$ & & 1.857 & 0.444 & & \\
Ranking results & & $A_{1} \sim A_{2}$ & $A_{1} \sim A_{2}<A_{3}$ & $A_{1}<A_{2} \sim A_{3}$ & $A_{1}<A_{2}$ & $A_{2}<A_{1}$ \\
\hline Abbasbandy, $p=1,2006$ & $A_{1}$ & 3 & 8 & 8 & 8 & 8 \\
& $A_{2}$ & 5 & 8.500 & 10 & 7.500 & 8.500 \\
Ranking results & $A_{3}$ & & 9.500 & 9 & & \\
\hline Abbasbandy, $p=2,2006$ & $A_{1}$ & 2.309 & 5.889 & 6.218 & 5.944 & 5.889 \\
& $A_{2}$ & 4.472 & 6.377 & 7.916 & 5.598 & 6.377 \\
Ranking results & $A_{3}$ & & 6.831 & 7.257 & & \\
\hline Cheng.1998 & $A_{1}<A_{2}$ & $A_{1}<A_{2}<A_{3}$ & $A_{1}<A_{3}<A_{2}$ & $A_{2}<A_{1}$ & $A_{1}<A_{2}$ \\
\hline Ranking results & $A_{1}$ & 1.725 & 4.031 & 4.358 & 3.707 & 4.031 \\
Chu.2002 & $A_{2}$ & 3.027 & 4.035 & 5.025 & 3.768 & 4.035 \\
& $A_{3}$ & & 4.694 & 5.020 & & \\
& & $A_{1}<A_{2}$ & $A_{1}<A_{2}<A_{3}$ & $A_{1}<A_{3}<A_{2}$ & $A_{1}<A_{2}$ & $A_{1}<A_{2}$ \\
\hline Ranking results & $A_{1}$ & 0.741 & 2 & 1.986 & 1.986 & 2 \\
Y. Deng.2006 & $A_{2}$ & 1.200 & 2.118 & 2.500 & 1.908 & 2.118 \\
& $A_{3}$ & & 2.374 & 2.222 & & \\
& & $A_{1}<A_{2}$ & $A_{1}<A_{2}<A_{3}$ & $A_{1}<A_{3}<A_{2}$ & $A_{2}<A_{1}$ & $A_{1}<A_{2}$ \\
\hline Ranking results & $A_{1}$ & 0.707 & 1.667 & 1.850 & 1.546 & 1.667 \\
Cheng.1998 & $A_{2}$ & 1.354 & 1.690 & 2.850 & 2.086 & 1.690 \\
& $A_{3}$ & & 1.922 & 2.167 & & \\
& & $A_{1}<A_{2}$ & $A_{1}<A_{2}<A_{3}$ & $A_{1}<A_{3}<A_{2}$ & $A_{1}<A_{2}$ & $A_{1}<A_{2}$ \\
\hline Panking results & $A_{1}$ & 0.133 & 0.167 & 0.397 & 0.242 & 0.167 \\
& $A_{2}$ & 0.667 & 0.292 & 0.433 & 0.151 & 0.292 \\
& $A_{3}$ & & 0.083 & 0.630 & & \\
& & $A_{2}<A_{1}$ & $A_{2}<A_{1}<A_{3}$ & $A_{3}<A_{2}<A_{1}$ & $A_{1}<A_{2}$ & $A_{2}<A_{1}$ \\
\hline & $A_{1}, A_{2}, A_{3}$ & $A_{1}<A_{2}$ & $A_{1}<A_{2}<A_{3}$ & $A_{2}<A_{3}<A_{1}$ & $A_{2}<A_{1}$ & $A_{1}<A_{2}$ \\
\hline
\end{tabular}




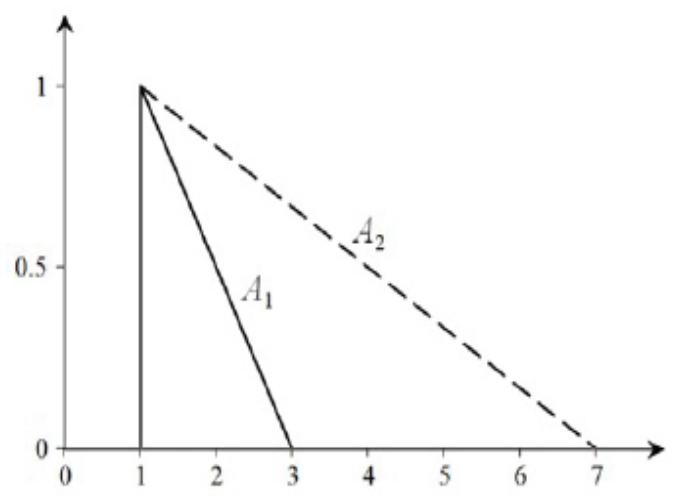

Figure 1: Set 1 of Example 8.

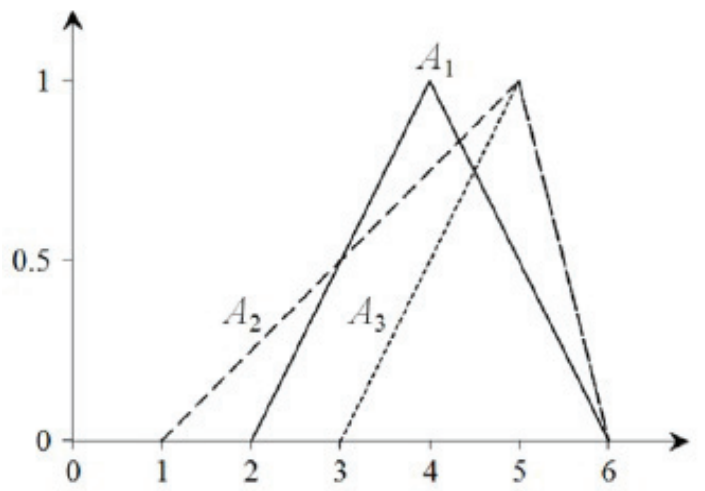

Figure 2: Set 2 of Example 8.

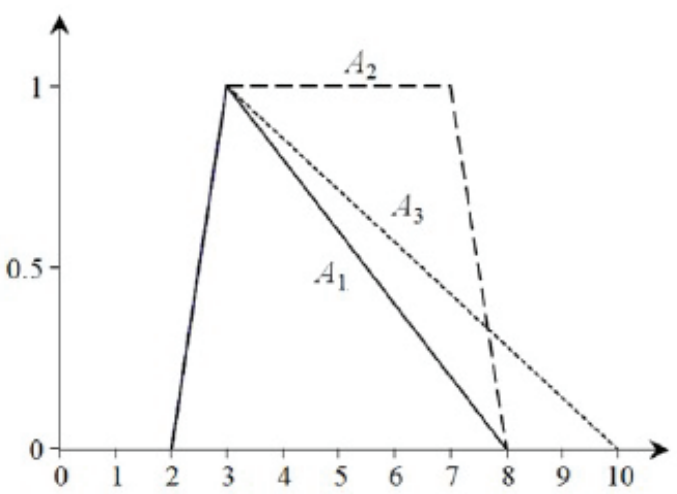

Figure 3: Set 3 of Example 8. 


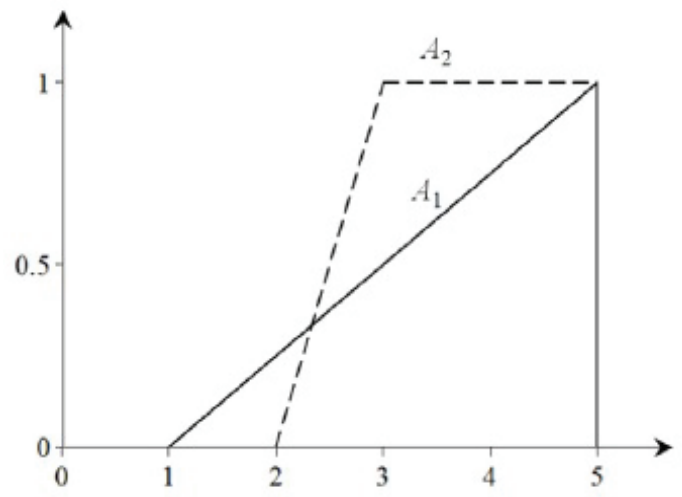

Figure 4: Set 4 of Example 8.

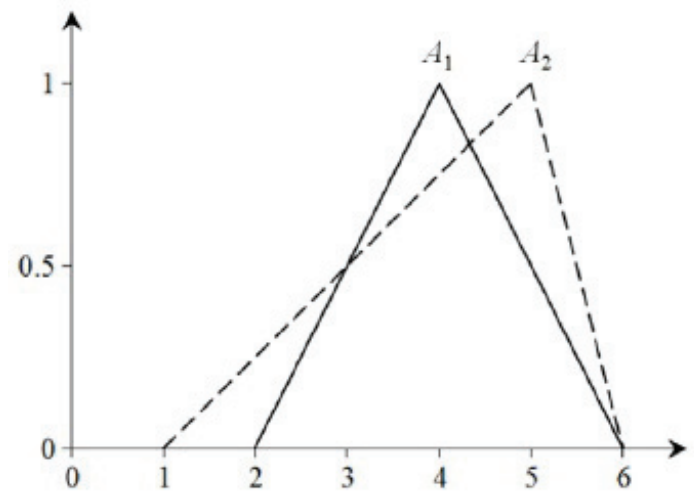

Figure 5: Set 5 of Example 8. 